\title{
JPEG Multi-Resolution Decomposition of Image Compression using Integer Wavelets
}

\author{
Vaibhav Raman \\ Department of CSE \\ ASET, Amity University, Noida
}

\author{
Richa Gupta \\ Department of CSE \\ ASET, Amity University, Noida
}

\begin{abstract}
To reduce the redundancy of image, image compression is used in order to store data in much more effective way. For effective \& efficient image compression integer wavelet transform has been introduced. The aim of the integer wavelet transform is to get maximum compression ratio possible. Decomposition of image takes place up to certain levels; at different levels the value of image compression is different. Maximum compression \& minimum compression can be achieved by integer wavelet transform method.
\end{abstract}

\section{Keywords}

Integer wavelets transform, Predictive Coding, Compression ratio.

\section{INTRODUCTION}

Image Compression is achieved by removal of one or more redundancy method :a) Coding Redundancy, which present when less than optimal code words are used i.e. for small length b) Interpixel, results from correlation between pixels of an image. Compression is important to manage the large amount of storage of data on internet. The types of image compression are lossless and lossy compression. JPEG compression can be used as both i.e. (as lossless or lossy) depending upon the condition. Lot of research has been made and also many researches are going on in lossless image compression.

The performance of Huffman Encoding \& Thresholding [12] and by $2 \mathrm{D}$ wavelet analysis was low \& does not allow flexibility of code stream, to overcome the problem Jpeg has been proposed. The technique performs compression based on Integer Wavelet filter.

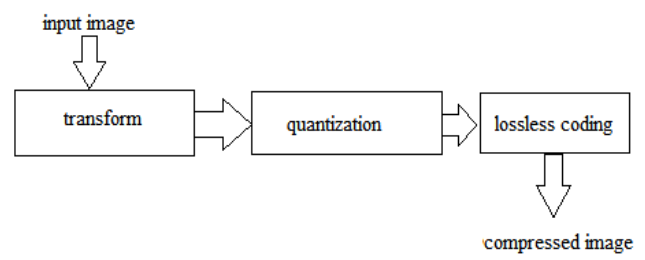

Fig 1: Image Compression System

In system, wavelet transform [2] method is used in Compression of the image. The Compressed image is decompressed by the wavelet transform in multi way. The encoding is done based on neighboring pixel value. Final image obtained in this method is a bit stream image which is binary. Receiver decodes incoming bit stream value, decompress it $\&$ reconstruct the original image.

\section{LITERATURE REVIEW}

Lossy spatial domain methods, exploit spatial redundancy in an image. Some information of the image is lost in the lossy method which can be compromised to get higher compression ratio. Mahmud Hasan [13] presented a method of spatial domain lossless image compression method. First step is that of each block all bits are reset, in the process $\mathrm{m} \times \mathrm{n}$ non overlapping block of image is done. The difference of min and max value are taken in these blocks, even though the method given reduces the good number of bits but only $13.77 \%$ result can only be obtained by the simple overhead of the bits. As in the method the author succeeds to increase the compression ratio but obtained compression ratio was low.

Amina khatun [1] given a model for the image compression that works on digital image and use of DCT image compression scheme has been proposed. The entropy coding is used that means level of decomposition of image can be done to a certain extend the author succeeds to get the compression ratio and MSE but the result obtained was low and for further implementation of the particular method the author has given that the implementation of it could also be done in neural networks.

Pallavi M. sune [11] presented an image compression method based on wavelet and Huffman coding. In this technique gray level value of image is stored and calculation of compression ratio and performance measures is done. Size of image is $n$ then image is multiplied by n. After multiplying, size will be smaller, the technique is relatively complex. The formula used by author is as;

M.S.E. = sum $($ sum $($ squared Error Image $)) /$ (rows * columns);

$\operatorname{PSNR}=10 * \log 10\left(255^{\wedge} 2 /\right.$ M.S.E $)$

Unit of PSNR is $\mathrm{db}$ and it gives the quality measure of the image obtained, and MSE is also obtained, smaller MSE means the minimum error and higher compression ratio means the image recovered is of better quality. In this method the use of Huffman coding results in satisfactory work.

M.y. Sanavuallah [14] proposed simple 2D - haar wavelet transformation in image compression, the aim is to minimize the computational by applying threshold for functions of wavelet and the quality of reconstructed image improved but author succeeds to obtain low quality image without any noticeable changes.

Ms S.S. Tamboli [15], proposed an image compression method by use of DWT, the main focus on the improvement of compression ratio and image quality. This is a technique achieved by wavelet which provides both the frequency and spatial information. Here the use of DWT with Haar wavelet occurs which helps in higher compression ratio. DWT performs compression of image signal and good quality image is obtained.

V. V. Sunil kumar [9] has proposed a technique regarding techniques by the use of wavelet transform, both the lossy and 
lossless compression is used the implementation of compression using wavelet transform by the use of EZW helps in the better result of compression ratio and also increases the performance measures. It works on both the spatial domain as well as in frequency domain.

Lossy spatial domain coding methods exploit the spatial redundancy in an image. In Lossy coding, the loss of information occurs and it comprised to get higher compression ratio, the compressed image cannot be perfectly reconstructed due to loss of information.

\section{IMAGE COMPRESSION MODEL}

By Image Compression Model, the Encoding and Decoding is defined. The compression method is divided into two parts: Encoder \& Decoder.

\subsection{Encoder}

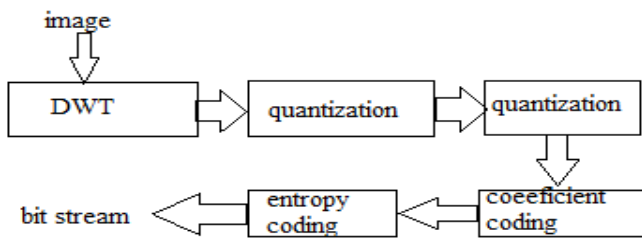

Fig 2: Encoding

\subsection{Decoder}

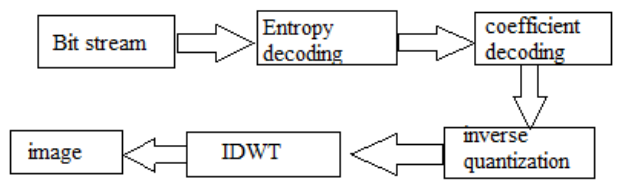

Fig 3: Decoding

The firstly encoding is applied and after that decoding is followed. In encoding, firstly image is input and it goes through transform coding by DWT, after that the process of quantization and coefficient is followed; the transform coefficients can be considered as a collection of quad-trees or zero-trees. Entropy method is the method that is capable of compressing it; here it is integer wavelet transform.

\section{INTEGER WAVELET TRANSFORM}

Wavelet transform [12] produces Floating point Coefficient. Original integer data can be reconstructed by using floating point coefficient.

We generally use floating point method because it is easy to implement as compared to others. Without any loss the reversible IWT [8](integer wavelet transform) which map integer pixel to its coefficient. Wavelet transform have really the best and efficient for transform based image compression. The coefficient of wavelet transform generally is 0 and 1 , that's why these are easy to code. In IWT, use of lifting is done in the spatial domain.

In the wavelet decomposition method [9] filtering method can be used. The filter length is same in any of the filter used. In this decomposition, DWT image is split into several sub bands (LL, LH, HL, and HH). The wavelet transform (WT), in general, produces the coefficients which are floating in number. The coefficients are used to reconstruct the perfect image using quantization results in a lossy scheme. The analysis filter bank decomposes the input signal into $2 \mathrm{sub}$ bands of signals.

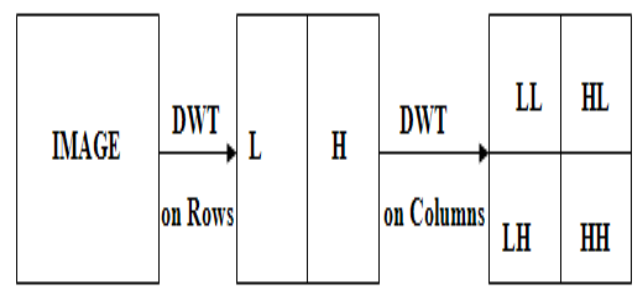

Fig 4: DWT on Rows \& Columns

The low frequency portion c (n) is given by c (n), where as the high frequency portion is denoted by $\mathrm{d}(\mathrm{n})$. Analysis part of filter is given by $x(n)$, where band is divided into the filters used which may be low pass or may be high pass. Af1 denotes the low pass filter where as af 2 represents high pass filter. After that each filter is down sampled by 2 . The main goal of the Integer wavelet transform [5] is to represent a function $f(x)$ as a linear combination of a set of such wavelets or basis functions which will be arbitrary. These functions can be obtained from scaling and shifting. In integer wavelet transform there are many functions of scaling present. The Coefficients value of integer wavelets are on the basis of the process of filtering. And the sample processes applied will the down sampling method. The steps involved in the wavelet transform, can be given by the two following equations i.e.

$$
\psi_{m, n}(t)=2^{m / 2} \psi\left(2^{m} t-n\right) ; \mathrm{m}, \mathrm{n} \in \mathrm{Z} \text { such that }-\infty<
$$

$\mathrm{m}, \mathrm{n}<\infty$

The Discrete wavelet transform can be given as the multiplication of the signals those are inside and these signals changed on the basis of the functions which are discrete in nature.

$$
\boldsymbol{W}_{m, n}=\left\langle x(t), \boldsymbol{\psi}_{m, n}(t)\right\rangle ; \quad \mathrm{m}, \mathrm{n} € \square Z ; \quad \mathrm{m}, \mathrm{n} \in \square Z
$$

The 1-D inverse DWT is given as:

$$
x(t)=\sum_{m} \sum_{n} W_{m, n} \psi_{m, n}(t) ; \quad \mathrm{m}, \quad \mathrm{n} \quad \in \square Z
$$

\section{SCHEME USED FOR METHODOLOGY}

The Scheme used for designing wavelet [10] is lifting scheme. In this scheme, all construction is derived from spatial domain. To calculate wavelet transforms it is the simple and effective method. It mainly consists of 3 steps: a) Split phase b) Predict phase c) Update phase. Basically, in split phase the data is split into 2 sets odd and even. In predict, odd set is predicted by even. And in update set, using wavelet coefficient updating of even set takes place and scaling function is calculated. 
Here in this scheme the implementation of reversible IWT takes place. In case of loss less compression perfect reconstruction can be done. Lifting scheme uses haar wavelet transform and used for speed of computation. Computation of scaling coefficient [9] requires adding 2 samples or dividing 2 samples. Calculation of wavelet coefficient requires subtracting 2 and dividing 2 samples. Example of Haar wavelet:

a. Consider sequence $\mathrm{f}(\mathrm{x})$ obtained by sampling.

b. Considering neighboring samples $\mathrm{a}$ and $\mathrm{b}$ of this sequence.

Strong correlation between two samples Haar wavelet transform replaces value as:

$\mathrm{a}=(\mathrm{A}+\mathrm{B}) / 2 \quad, \quad \mathrm{~d}=(\mathrm{B}-\mathrm{A}) / 2$

Inverse Haar transform can be calculated as:

$A=(a-d / 2) \quad, \quad B=(a+d / 2)$

\subsection{Forward Lifting Scheme}

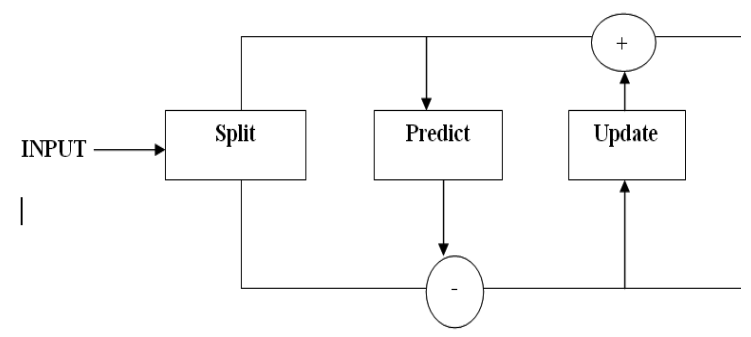

Fig 5: Forward Lifting Scheme

\subsection{Inverse Lifting Scheme}

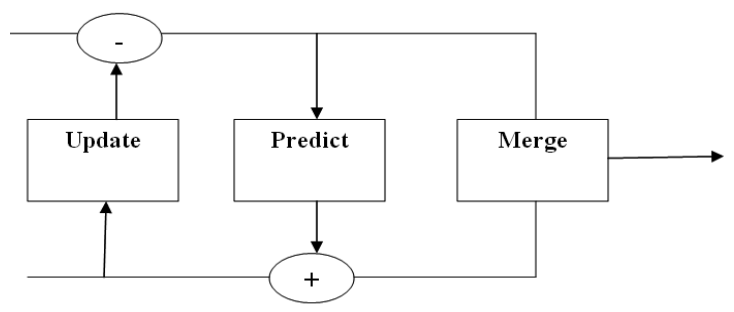

Fig 6: Forward Lifting Scheme

In this scheme, all construction is derived from spatial domain. To calculate wavelet transforms it is the simple and effective method. It mainly consists of 3 steps: a) Split phase b) Predict phase c) Update phase. Basically, in split phase the data is split into 2 sets odd and even. In predict, odd set is predicted by even. And in update set, using wavelet coefficient updating of even set takes place and scaling function is calculated. In decomposition image is pass by a low pass and band pass filter and down sample by factor 2 . And the process repeats until 4 outputs.

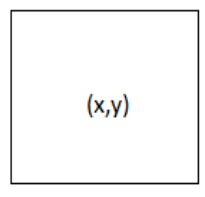

Input Image

\begin{tabular}{|c|c|}
\hline LL & LH \\
\hline HL & HH \\
\hline
\end{tabular}

Output

Image
Fig 7: Decomposition of Image

\section{IMPLEMENTATION}

It involves reduction of filter coefficient by factor of $3 / 2$ and also predictive coding [8] is involved. Haar filter in lifting scheme [8] and filter coefficient is given as;

$$
\begin{aligned}
& X 1=\left[\begin{array}{llll}
-1 & 9 & 9 & 1
\end{array}\right] / 16 ; \\
& X 2=\left[\begin{array}{llll}
0 & 0 & 1 & 1
\end{array}\right] /(-4) ;
\end{aligned}
$$

$\mathrm{X} 1$ is prediction filter coefficient.

$\mathrm{X} 2$ is update filter coefficient.

Reduced filter coefficient is given by;

$$
\begin{aligned}
& X 1=\left[\begin{array}{llll}
-1 & 9 & 9 & 1
\end{array}\right] /\left(16^{*} 1.5\right) ; \\
& X 2=\left[\begin{array}{llll}
0 & 0 & 1 & 1
\end{array}\right] /(-4 * 1.5) ;
\end{aligned}
$$

$f(n)$

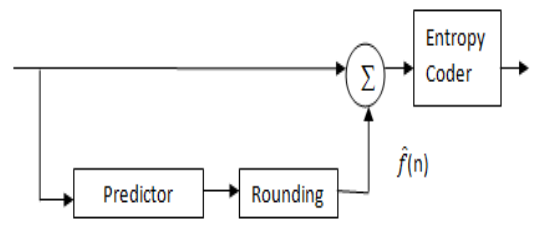

Predictive encoder
Fig 8:

Predictive Coding [8] Techniques are used to perform lossless Image compression.

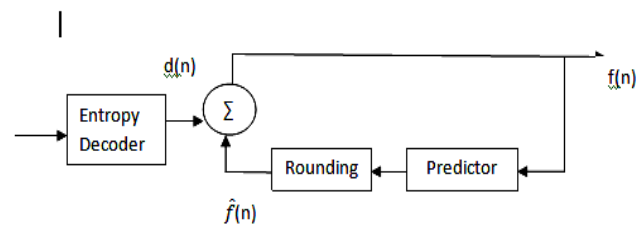

Fig 9: Predictive Decoder

In this method IWT [12] is applied on the image which divides the image into four sub band. Now predictive coding is applied on the four different bands separately giving 4 outputs. The reconstruction processes involves applying the predictive decoding followed by inverse integer transform.

\subsection{Experimental Results}

Table of Compression Ratio (\%) Obtained and time of execution in (seconds) at different level 
Table 1 Comparisons of CR \& Execution time

\begin{tabular}{|l|l|l|}
\hline At level 1 & $51.254 \%$ & 9.72 \\
\hline At level 2 & $84.5457 \%$ & 8.03 \\
\hline At level 3 & $95.593 \%$ & 5.48 \\
\hline At level 4 & $98.7933 \%$ & 4.66 \\
\hline At level 5 & $99.6742 \%$ & 4.92 \\
\hline
\end{tabular}

\begin{tabular}{|l|l|l|}
\hline At level 6 & $99.9102 \%$ & 6.18 \\
\hline At level 7 & $99.9741 \%$ & 6.16 \\
\hline At level 8 & $99.9934 \%$ & 4.60 \\
\hline At level 9 & $99.9973 \%$ & 5.81 \\
\hline At level 10 & $99.9994 \%$ & 6.28 \\
\hline
\end{tabular}

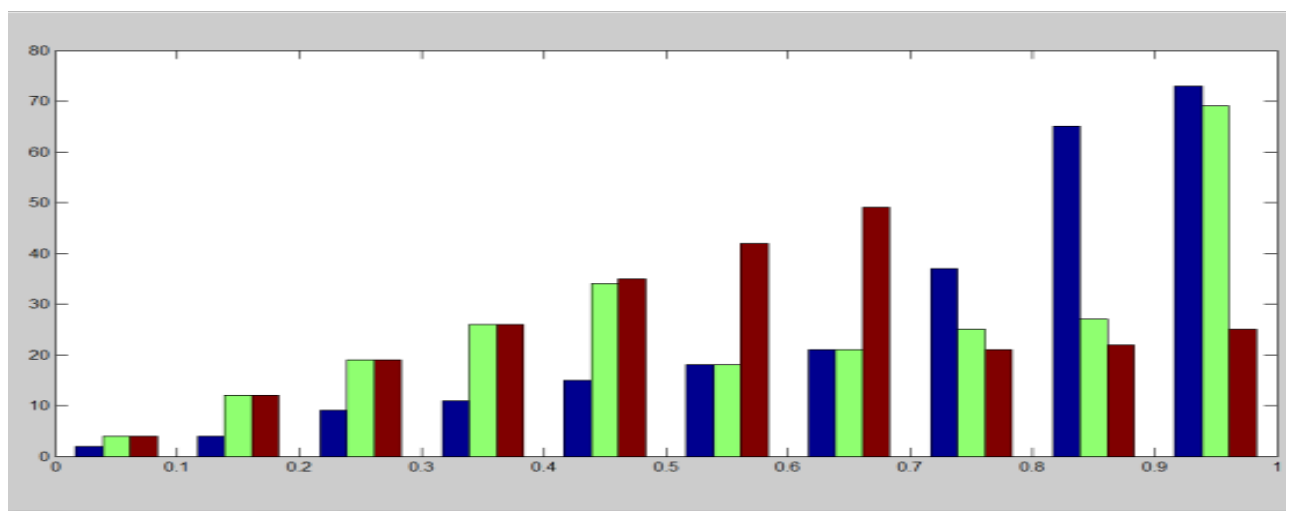

Graph showing Comparisons of original image, compressed image and reconstructed image by sample values of entropy coding \& predictive coding

\section{CONCLUSION}

This method betters the quality on Compression Ratio (CR), speed of image compression also increases. Maximum compression ratio is gained by using multi level decomposition using integer wavelet transform. The problem of limitation in format of image types like .png ,.bmp etc is solved and the Image compression for JPEG types of file can also be done by using this method ,the limitation in Gaussian ,Haar Wavlet method is sorted out here by using this approach. This approach have less complexity and less computational than other techniques that are used in previous approaches the Integer multi wavelet transform successfully solve the limitations of CR, and have better quality of image compression.

\section{ACKNOWLEDGMENT}

I would like to express my gratitude to Ms. Richa Gupta and Mr. Abhishek Singhal, Faculty Computer Science \& Engineering Department Amity University, for their guidance, proofreading and help in completing this work successfully.

\section{REFERENCES}

[1] M. Mozammel Hoque Chowdhary \& Amina Khatun "Image Compression Using Discrete Wavelet Transform” IJCSA, Volume 9, Issue 4, No1, July 2012.

[2] Michael B.Martin and Amy E.Bell, "New Image Compression Techniques using Multi wavelets and Multi wavelet packets" $\|$ IEEE Transactions on Image Processing, vol.10, No.4, April 2007, pp.500-511.

[3] T.Acharya and Ping-Sing Tsai, "JPEG2000 Standard for Image Compression Concepts, algorithms and VLSI Architectures" John \& Sons press, 2005.

[4] E.farzad, C.Matthieu, and W.stefan, "JPEG versus JPEG2000: an objective comparison of image encoding quality," SPIE.

[5] T Hong LIU , Lin-pei ZHAIV , Ying GAO, Wen-ming $\mathrm{LI}^{\mathrm{e}}$, Jiu-fei $\mathrm{ZHOU}{ }^{\mathrm{ee}}$, "Image Compression Based on
Biorthogonal Wavelet Transform", IEEE Proceedings of ISCIT, 2005.

[6] "A Comparative Study of Image Compression Techniques Based on Svd, D,wt-Svt , Dwt-Dct" ICSCI2008 proceedings pg 494-496.

[7].Padmaja.V.K Dr. B. Chandrasekhar, "Literature Review of Image Compression Algorithm" IJSER, volume 3, Issue 6, June 2012.

[8] Vineeta Gupta, A.G. Rao \& Krishna Mohan Pandey "Integer Wavelet Transform \& Predictive Coding For Lossless Medical Image Compression” IJET volume 2 Issue 4, August 2013.

[9] Image Compression Techniques by using Wavelet Transform V. V. Sunil Kumar1* M. Indra Sena Reddy2 Vol 2, No.5, 2012.

[10] COMPRESSION METHODS USING WAVELET TRANSFORM ,Ms S. S.Tamboli, June (2012).

[11] Ms. Pallavi M. sune and Prof. Vijaya K. Shandilya "Image Compression technique based on wavelet \& Huffman coding" IJARCSSE, volume 3, issue 4, April 2013.

[12] Mr. E. Praveen, Dr. M.G. Sumithra"Medical Image Compression Using Integer Multi Wavelet Transform for Telemedicine application" IJECS volume 2 Issue 5 may, 2013, page no. 1663-1669.s.

[13] Mahmud Hasan "An improved Approach International Journal of Scientific \& Engineering Research, Volume 3, Issue 4, April-2012.

[14] P. Raviraj and M.Y. Sanavullah" The Modified 2D-Haar Wavelet Transformation in Image Compression" IDOSI Publications Journal of Scientific Research issue2, volume2, 2007.

[15] Ms S. S. Tamboli “COMPRESSION METHODS USING WAVELET TRANSFORM” IJCET Volume 3, Issue 1, January- June (2012). 